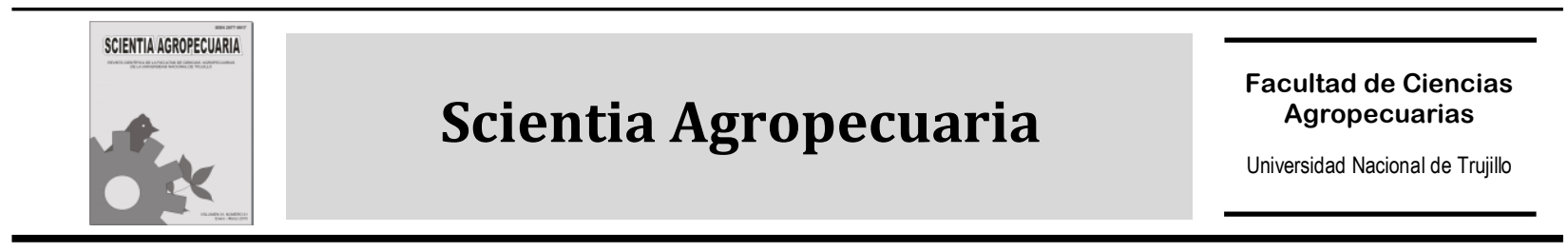

\title{
Estudio comparativo de la pérdida de vitamina $C$ en chalarina (Casimiroa edulis) por cuatro métodos de deshidratación
}

\section{Comparative study of the loss of vitamin $\mathrm{C}$ in chalarina (Casimiroa edulis) by four methods of dehydration}

\author{
Judith Castañeda ${ }^{1, *}$, Hubert Arteaga $^{2}$, Raúl Siche $^{2}$, Gilbert Rodriguez $^{3}$ \\ ${ }^{1}$ Empresa Perú Spices S.A. Calle Camino Real s/n. Mz I Lt 1Z. Ind Miramar-Moche, Trujillo (Perú). \\ ${ }^{2}$ Departamento de Ciencias Agroindustriales (Universidad Nacional de Trujillo) Av. Juan Pablo II s/n, Trujillo, Perú \\ ${ }^{3}$ Escuela de Ingeniería Agroindustrial (Universidad Nacional del Santa), Av. Universitaria s/n. Urb. Bellamar, Chimbote, Perú
}

Recibido 02 febrero 2010; aceptado 30 marzo 2010

\begin{abstract}
Resumen
El objetivo de este estudio fue comparar la pérdida de vitamina $\mathrm{C}$ de la chalarina después de aplicar cuatro métodos de deshidratación: deshidratación osmótica a vacío combinada con secado convectivo, secado convectivo, deshidratación osmótica a vacío combinada con liofilización, y liofilización. La chalarina fresca fue acondicionada en forma de cubos de $1 \mathrm{~cm}$ de arista; se determinó el contenido de vitamina $\mathrm{C}$ y otras propiedades químicas de la pulpa. Los polvos obtenidos fueron caracterizados: contenido de vitamina $\mathrm{C}$, contenido de humedad, $\mathrm{a}_{\mathrm{w}}$, densidad, ángulo de fluencia y compresibilidad. La pulpa de chalarina fresca presentó un contenido de vitamina $\mathrm{C}$ de 29.75 $\mathrm{mg} / 100 \mathrm{~g}$. La deshidratación osmótica a vacío redujo este contenido hasta $15.75 \mathrm{mg} / 100 \mathrm{~g}$, que combinado con secado convectivo se llega a $9.31 \mathrm{mg} / 100 \mathrm{~g}$; en tanto, combinado con liofilización se obtuvo $14.98 \mathrm{mg} / 100 \mathrm{~g}$. Aplicando sólo secado convectivo se obtuvo $7.05 \mathrm{mg}$ de vitaminaC/100g de polvo de chalarina, mientras que aplicando sólo liofilización se obtuvo $23.63 \mathrm{mg} / 100 \mathrm{~g}$. Se concluye que el polvo obtenido por deshidratación osmótica a vacío secado convectivo presenta mejores características fisicoquímicas que los polvos obtenidos con los otros métodos de deshidratación.
\end{abstract}

Palabras clave: Chalarina, vitamina C, deshidratación osmótica, liofilización

\begin{abstract}
The aim of this study was to compare the loss of chalarina vitamin $\mathrm{C}$ after applying four methods of dehydration: vacuum osmotic dehydration combined with convective drying, convective drying, osmotic dehydration combined with vacuum freeze-drying, and freeze-drying. The fresh chalarina was fitted into cubes of $1 \mathrm{~cm}$; we determined the vitamin $\mathrm{C}$ content and other chemical properties of the pulp. The powders were characterized: vitamin $\mathrm{C}$, moisture content, $\mathrm{a}_{\mathrm{w}}$, density, angle of flow and compressibility. Fresh chalarina pulp has a vitamin $\mathrm{C}$ content of 29.75 $\mathrm{mg} / 100 \mathrm{~g}$. Osmotic dehydration and vacuum reduced to $15.75 \mathrm{mg} / 100 \mathrm{~g}$ this content; combined with convective drying is reached $9.31 \mathrm{mg} / 100 \mathrm{~g}$; if combined with freeze-drying is obtained $14.98 \mathrm{mg} / 100 \mathrm{~g}$. Applying convective drying was obtained only $7.05 \mathrm{mg}$ of vitaminaC/100g chalarina powder, while by applying only freeze-drying was obtained $23.63 \mathrm{mg} / 100 \mathrm{~g}$. We conclude that the powder obtained by vacuum osmotic dehydration - convective drying has better physical and chemical characteristics of powders obtained with other methods of dehydration.
\end{abstract}

Keywords: Chalarina, vitamin C, osmotic dehydration, freeze-drying

\section{Introducción}

En la actualidad, las tendencias en la alimentación han dado un giro hacia lo natural y autóctono buscando productos saludables, nutritivos y de fácil preparación; productos con un mínimo tratamiento y con

\footnotetext{
* Autor para correspondencia.

E-mail: qualitycontrol@ peruspices.com, juvi366@hotmail.com (J. Castañeda)
} 
menor agregado de ingredientes sintéticos, de tal modo que estos contribuyan a preservar la salud y hasta curar (Boucher, 1999).

El Perú produce gran diversidad de frutas, que por sus características exóticas de aroma, sabor y contenidos nutricionales, son muy apreciados en los mercados internacionales, donde adquieren valores muy altos, comparado con los precios de comercialización de estas frutas en los mercados nacionales. Adicionalmente, el auge agroexportador del Perú, lleva a buscar tendencias exportadoras modernas, como la exportación de frutos poco difundidos. Uno de estos productos es la chalarina (zapote blanco); sin embargo, la chalarina por ser un fruto perecible, es susceptible a grandes pérdidas en postcosecha debido a sus características fisiológicas tan particulares. Una alternativa para la chalarina, consiste en deshidratarlas osmóticamente y someterlas a secado convectivo o liofilización.

\section{Materiales y métodos}

Se utilizó Chalarina (Casimiroa edulis Llave), producida en el Valle de Condebamba provincia de Cajabamba en La Libertad.
Recepcionada la materia prima, fue seleccionada, lavada, pelada, despepada y troceada en forma de cubos de $1 \mathrm{~cm}$ de arista. A los cubos frescos de chalarina se les determinó el contenido de vitamina $\mathrm{C}$ y otras características químicas de importancia (Figura 1).

Posteriormente los cubos fueron sometidos a cuatro métodos de deshidratación: a) Deshidratación osmótica a vacio liofilización; b) Deshidratación osmótica a vacío - secado convectivo; c) Liofilización; y, d) secado convectivo. Para la deshidratación osmótica se utilizó una concentración de sacarosa de $63.57 \% \mathrm{~m} / \mathrm{m}$. Además, la operación fue llevada a cabo con $679.06 \mathrm{~mm}$ $\mathrm{Hg}$ de presión de vacío y $45.3^{\circ} \mathrm{C}$ de temperatura. Para el secado convectivo fue utilizado $3.02 \mathrm{~m} / \mathrm{s}$ de velocidad de aire y $69.5^{\circ} \mathrm{C}$ de temperatura de aire a la entrada de la cámara de secado. La liofilización fue llevada con $1000 \mathrm{umHg}$ de presión y $-40^{\circ} \mathrm{C}$ de temperatura en la cámara. La chalarina deshidratada, en todos los casos, fue molida con la finalidad de homogenizar el polvo, Al polvo obtenido con cada método de secado, se le determinó el contenido de vitamina $\mathrm{C}$ y otras características físicas (Figura 1).

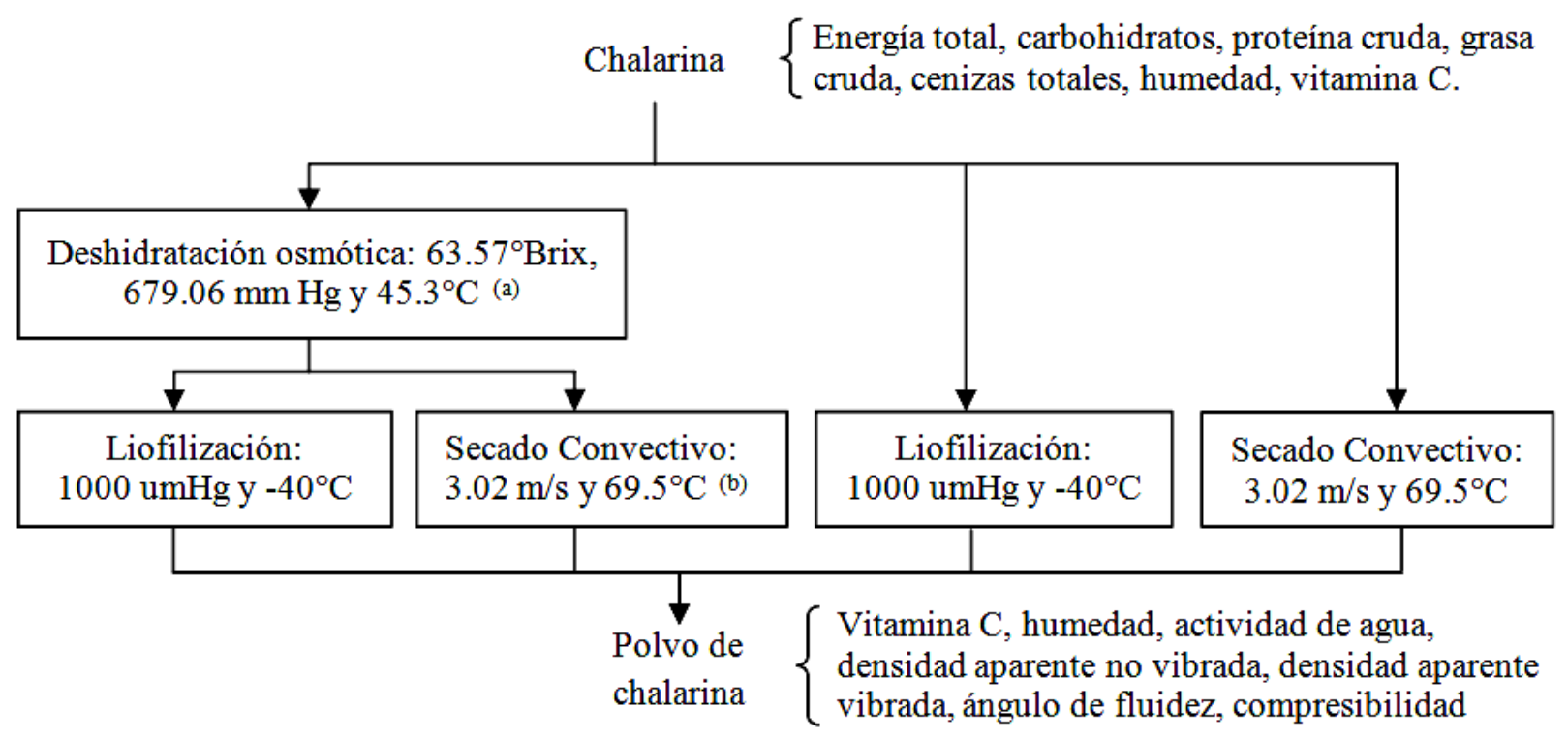

Figura 1. Esquema experimental para la deshidratación de chalarina por diferentes métodos. 


\section{Análisis físicos y químicos en chalarina fresca $y$ deshidratada}

Para la chalarina fresca se realizaron análisis de humedad (método de secado en estufa), proteínas (método de Kjeldahl), grasa cruda (método soxlet), cenizas (incineración directa), carbohidratos (por diferencia), y vitamina $\mathrm{C}$ (titulación con 2,6 diclorofenol indofenol). Para la chalarina deshidratada se realizaron análisis de humedad y vitamina $\mathrm{C}$ (métodos indicados anteriormente), actividad de agua (isotermas de adsorción a través de los métodos BET y GAB), densidad aparente no vibrada y densidad aparente vibrada (Alvarado y Aguilera, 1994), ángulo de reposo (método de inyección, Alvarado y Aguilera, 1994), y compresibilidad (relación de densidades aparentes del polvo sin vibrar y vibrado).

\section{Resultados y discusión}

\section{Caracterización de la chalarina fresca}

En la Tabla 1 se muestra la composición química de la pulpa de chalarina fresca utilizada, así como los valores teóricos reportados para este producto.

\section{Tabla 1}

Composición química de la chalarina fresca (100 g parte comestible)

\begin{tabular}{lcc}
\hline Característica & $\begin{array}{c}\text { Valor } \\
\text { obtenido }^{(a)}\end{array}$ & $\begin{array}{c}\text { Valor } \\
\text { teórico }^{(b)}\end{array}$ \\
\hline Carbohidratos $(\mathrm{g})$ & 16.9 & 15.7 \\
Proteína cruda (g) & 1.3 & 1.4 \\
Grasa cruda (g) & 0.0 & 0.4 \\
Cenizas totales (g) & 0.5 & 0.5 \\
Humedad (g) & 81.3 & 82.0 \\
Vitamina C (mg) & 29.75 & 23.0 \\
\hline
\end{tabular}

(a) La Molina Calidad Total Laboratorios-UNALM

(b) Menchu y Méndez (2008).

Se observa que los valores obtenidos aquí son semejantes a los reportados por Menchu y Méndez (2008). La diferencia más notoria es justamente en la vitamina $\mathrm{C}$, que puede deberse al origen de la fruta, pues los valores reportados por Menchu y Méndez (2008) corresponden a frutos de procedencia centroamericana, lugar donde las condiciones ambientales y edafológicas son distintas a las del valle de Condebamba. Arcila (2002) precisa que la actividad fisiológica y el desempeño productivo de una especie vegetal dependen de la interacción armónica entre su genotipo y los factores ambientales, pudiendo variar bajo diferentes condiciones edafológicas y climáticas. La fruta fresca presenta valores elevados de humedad $(81.3 \%)$, que lo hace muy susceptible al deterioro por invasión microbiana.

La vitamina $\mathrm{C}$ es un compuesto muy utilizado en la tecnología de alimentos como índice de calidad nutricional de productos alimenticios. Ceballos (2008) precisa que la vitamina C ayuda a mantener el colágeno, disminuir los daños por estrés, es un antioxidante $\mathrm{y}$ participa en la biosíntesis de aminoácidos. Además indica que esta vitamina es la vitamina más sensible que se encuentran en los alimentos y su estabilidad varía en función de las condiciones ambientales tales como el $\mathrm{pH}$, la concentración de trazas de iones metálicos y oxígeno, la temperatura, el tipo de proceso de deshidratación precisando que la mayor retención se da a bajas temperaturas y cortos tiempos. Al respecto Cevallos y Velásquez (2007) agregan que si esta vitamina resiste los tratamientos térmicos de los alimentos, todos los demás nutrientes se encuentran en buen estado. En este trabajo, se encontró en la chalarina fresca tuvo 29.75 mg/100 g de acido ascórbico (Tabla 1), valor que fue tomado como referencia para evaluar el efecto de los métodos de deshidratación. Chizmar (2009) indica que el fruto Casimiroa edulis Llave es rico en vitaminas A y C, y posee un alto contenido de carbohidratos $\mathrm{y}$ proteínas.

\section{Caracterización del polvo de chalarina}

En la Tabla 2 se presentan los resultados de los ensayos realizados al polvo chalarina, obtenidos por los diferentes métodos de deshidratación. 
Tabla 2

Características fisicoquímicas del polvo de chalarina obtenido por cuatro métodos de deshidratación

\begin{tabular}{lcccc}
\hline Características & DOV $+\mathrm{SC}$ & $\mathrm{SC}$ & $\begin{array}{c}\text { DOV }+ \\
\text { Liofilización }\end{array}$ & Liofilización \\
\hline Vitamina C $(\mathrm{mg} / 100 \mathrm{~g}$ de producto reconstituido) & 9.313 & 7.052 & 14.985 & 23.634 \\
Humedad $(\%)$ & 10 & 10.03 & 1.82 & 2.02 \\
Actividad de agua $\left(\mathrm{a}_{\mathrm{w}}\right)$ & 0.6 & 0.33 & 0.023 & 0.015 \\
Densidad aparente no vibrada $\left(\mathrm{kg} / \mathrm{m}^{3}\right)$ & 705.6 & 671.6 & 543.5 & 505.9 \\
Densidad aparente vibrada $\left(\mathrm{kg} / \mathrm{m}^{3}\right)$ & 772.2 & 762.6 & 608 & 585 \\
Ángulo de fluidez $\left({ }^{\circ}\right)$ & 20.3 & 23.7 & 29.2 & 37.7 \\
Compresibilidad $(\%)$ & 8.62 & 11.93 & 10.61 & 13.52 \\
\hline
\end{tabular}

DOV: Deshidratación osmótica a vacío

SC: Secado convectivo

La DOV disminuyó el contenido de agua a 44.5\%. Por otro lado, se perdió el $47.05 \%$ de la vitamina C. Esta pérdida fue ocasionada, en menor incidencia, por la exposición de la fruta a altas temperaturas y presión de vacío. El factor de mayor incidencia sobre esta pérdida es la disolución acuosa en la que estaban sumergidos los trozos de fruta, ya que la vitamina C (vitamina hidrosoluble) se pierde por lixiviación (Badui, 1999). Badui (1999) también afirma que la degradación de la vitamina $\mathrm{C}$ en muestras pulpeadas se debe en gran parte a la oxidación que se presenta por la incorporación de aire durante el despulpado.

Considerando, además de la DOV, la aplicación de secado convectivo hasta una humedad final de $10 \%$ en base húmeda, se obtuvo un contenido de vitamina $\mathrm{C}$ de 9.313 $\mathrm{mg} / 100 \mathrm{~g}$ de producto reconstituido (Tabla 2). Este resultado representa una pérdida del $40.88 \%$ respecto a la chalarina osmodeshidratada y $68.69 \%$ respecto a la chalarina fresca (proceso combinado DOV+SC). Sidonia (2008) precisa que las pérdidas en Vitamina $\mathrm{C}$ pueden ser explicadas por el hecho que el ácido ascórbico es un componente muy sensible a la temperatura, por lo que se degrada por efecto del calor, ello, además del efecto degradativo producto de la oxidación. Lo indicado por Sindoni (2008) se pone de manifiesto, en mayor medida, al evaluar el tratamiento individual del secado convectivo en el que se obtuvo $7.052 \mathrm{mg} / 100 \mathrm{~g}$, equivalente a una pérdida de
76.29\% respecto de la chalarina fresca. Queda en evidencia que la degradación de la vitamina $\mathrm{C}$ es mayor en la medida que se incrementa el tiempo de exposición a factores oxidantes, como el oxígeno, y su efecto es aun mayor a temperaturas elevadas (Ramos, 2002).

El contenido de vitamina $\mathrm{C}$ tras el proceso de liofilización de la pulpa chalarina osmodeshidratada fue de $14.985 \mathrm{mg} / 100 \mathrm{~g}$ (DOV+Liofilización, Tabla 2). En este caso, se perdió solamente $4.87 \%$ de vitamina $\mathrm{C}$, respecto a la chalarina osmodeshidratada, pero se perdió $49.63 \%$ de vitamina C cuando se combinaron los procesos DOV y Liofilización.

La liofilización de fruta fresca reportó un polvo con un contenido de vitamina $\mathrm{C}$ de $23.634 \mathrm{mg} / 100 \mathrm{~g}$. Este proceso permite una pérdida de vitamina $\mathrm{C}$ de sólo $21.47 \%$, valor muy por debajo de los otros 3 métodos analizados. Ceballos (2008) menciona que la liofilización permite disminuir la pérdida de compuestos volátiles o termosensibles, como la vitamina $\mathrm{C}$.

Se obtuvo 0.6 de actividad de agua en el polvo obtenido (humedad alrededor del 10\%) con DOV+SC y 0.33 para secado convectivo. En el polvo obtenido con DOV+liofilizado se obtuvo una humedad del $1.82 \%$, correspondiéndole una actividad de agua de 0.023 ; y para la fruta liofilizada una humedad de $2.02 \%$, correspondiéndole una actividad de agua de 0.0015 . Se observa que al utilizar 
DOV, como proceso previo al secado convectivo y a la liofilización, se incrementa la actividad de agua respecto a los procesos únicos.

El polvo con mayor densidad $\left(705.6 \mathrm{~kg} / \mathrm{m}^{3}\right.$, Tabla 2) fue obtenido por el proceso combinado DOV+SC. Posiblemente, el ingreso de azúcar a la pulpa en la DOV, resulte en un aumento de la densidad y genere un polvo más compacto. La liofilización, por otro lado, promueve la unión de partículas, unas con otras, de manera aleatoria, resultando un agregado de estructura abierta y porosa de mayor tamaño que las partículas originales (Barbosa-Cánovas et al, 1997). Los alimentos en polvo que se utilizan como ingredientes en otros procesos se requiere posean una mayor densidad y mayor variabilidad en el tamaño de partículas (Barbosa et al, 1997).

Según Peleg y Pollak (1982) el ángulo de reposo para polvos no cohesivos es menor a $35^{\circ}$ y el ángulo de reposo de polvos cohesivos es mayor a $45^{\circ}$. Se puede afirmar que el polvo de chalarina obtenido por DOV+SC $\left(20.3^{\circ}\right.$ de ángulo de reposo, Tabla 2), fluye libremente; mientras que el polvo obtenido por liofilización (37. $7^{\circ}$ de ángulo de reposo, Tabla 2) se asemeja a una sustancia cohesiva, en las que se tiene que mejorar su fluidez. La compresibilidad de una masa pulverulenta está en relación inversa a su fluidez.

En las isotermas de adsorción de la Figura 2 se observa el comportamiento higroscópico del polvo en función del método de deshidratación empleado en la Chalarina. El polvo menos higroscópico fue obtenido por $\mathrm{DOV}+\mathrm{SC}$, el efecto opuesto se observa en el polvo obtenido de la pulpa liofilizada. Barbosa-Cánovas et al. (1997) asociaron la higroscopicidad del producto alimenticio con el tamaño del poro. La liofilización, al utilizar altas presiones de vacío, produce un polvo poco compacto con muchos poros, por eso muestra mayor higroscopicidad; mientras que al adicionar azúcar a la fruta en la deshidratación osmótica a vacio, se obtiene un polvo con una constitución más compacta y disminuye la higroscopicidad.

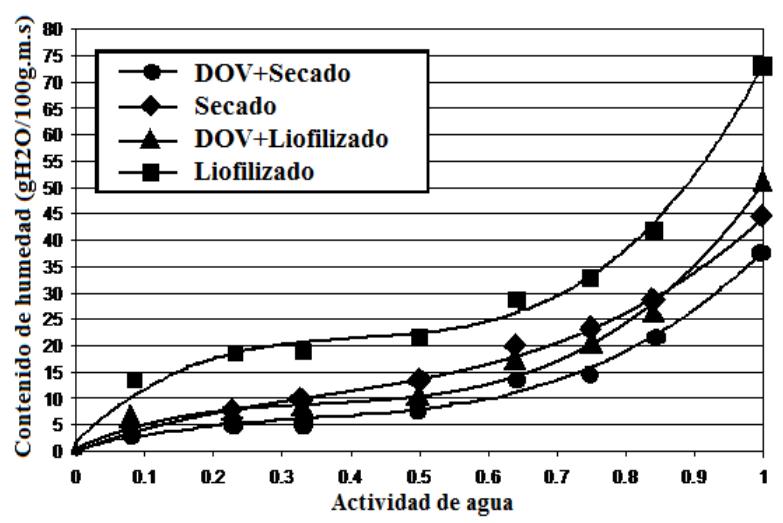

Figura 2. Isotermas de adsorción del polvo de chalarina obtenido por cuatro tratamientos de deshidratación.

\section{Conclusiones}

Los métodos de deshidratación estudiados reducen el contenido de vitamina $\mathrm{C}$ de la chalarina, siendo el secado convectivo el método que causa mayor pérdida de este nutriente $(76.26 \%)$; mientras que la liofilización es el método que causa menos pérdida $(21.47 \%)$. La DOV permite pérdidas del orden de $47.05 \%$, pero ayuda en la retención de este nutriente si se combina con secado convectivo. El polvo de chalarina obtenido por $\mathrm{DOV}+\mathrm{SC}$ presentan un mayor contenido de humedad, mayor $\mathrm{a}_{\mathrm{w}}$, es más denso, tienen mayor fluidez y es menos cohesivo que los obtenidos por liofilización.

\section{Referencias}

Alvarado, J.; Aguilera, J. 1994. Métodos para medir las propiedades físicas en industrias de alimentos. Editorial acribia, S.A. Zaragoza-España.

Arcila, P.; Giraldo, G.; Celis, F.; Duarte, J. 2002. Cambios físicos y químicos durante la maduración del plátano dominico-hartón (Musa AAB Simmonds) en la región cafetera central colombiana. ACORBAT Memorias XV reunión, Asociación de bananeros de Colombia AUGURA. Colombia. Disponible en: http://musalit.inibap.org/pdf/IN030078_es.pdf

Badui, S. 1999. Química de alimentos. $3^{\circ}$ edición, Editorial Pearson. México.

Barbosa-Cánovas, G.; Vega, H. 2002. Deshidratación de Alimentos. Editorial Acribia S.A. Zaragoza - España. 
Boucher, F. 1999. Los productos nutracéuticos oportunidades para los recursos naturales autóctonos el papel de los investigadores. Fascículo técnico $\mathrm{N}^{\circ} 18$. Instituto interamericano para la agricultura centro regional andino (IICA). Disponible en: http://www.iica.int/Esp/organizacion/LTGC/agroindustri a/Documentos\%20Agroindustria\%20Rural/nutraceuticos fb.pdf

Ceballos, A. 2008. Estudio comparativo de tres sistemas de secado para la producción de un polvo deshidratado de fruta. Tesis para obtener el grado de magister. Universidad Nacional de Colombia sede Manizales. Disponible en: http://digital.unal.edu.co/dspace/ bitstream/10245/1122/1/adelamariaceballospenaloza.200 8.pdf

Cevallos, R.; Velásquez, L. 2007. Comparación de la temperatura-tiempo de retención de pasteurización y su efecto en la concentración de vitamina $\mathrm{C}$ en el zumo de naranja. Tesis para optar el título de Ingeniero Agroindustrial. ESPAM.

Chizmar, C. 2009. Plantas comestibles de centroamerica. $1^{\circ}$ edición. Instituto Nacional de Biodiversidad INBio. Santo Domingo de Heredia- Costa Rica. Disponible en: http://www.inbio.ac.cr/web-ca/biodiversidad/regional/ PlantasComestiblesCA-VE.pdf

Menchu, T.; Méndez, H. 2007. Tabla de composición de alimentos de Centroamérica. $2^{\circ}$ edición, INCAP y OPS. Guatemala. Disponible en: http://new.paho.org/incap/ index 2.php?option=com_docman\&task $=$ doc_view\&gid= 80\&Itemid $=282$

Peleg, M.; Pollak, N. 1982. The problem of equilibrium conditions in stress relaxation analyses of solid food. Journal Texture Studies 13: 1 - 11 .

Ramos, Z. 2002. Evaluación de factores de procesamiento y conservación de pulpa de myrciaria dubia h.b.k. (camucamu) que reducen el contenido de vitamina $\mathrm{C}$ (ácido ascórbico). Revista amazónica de investigación alimentaria V2(2), 89-99p. Iquitos -Perú. Disponible en: http://www.unapiquitos.edu.pe/links/facultades/ alimentarias/v22/7.pdf

Sindoni, M. 2008. Efecto de la deshidratación sobre las características fisicoquímicas del merey (anacardium occidentale 1.). XX Congreso Brasilero de Fruticultura. Disponible en: http://200.137.78.15/cd_XXCBF/ paginas/ProcessAgro/20080730_161431.pdf 